Gilles Veinstein, dir., Syncrétismes et hérésies dans l'Orient seldjoukide et ottoman (xive-xviiie siècles)

Louvain, Peeters, coll. « Turcica » (XIV), 2005, 428 p.

\title{
Christian Décobert
}

\section{(2) OpenEdition}

\section{Journals}

Édition électronique

URL : http://journals.openedition.org/assr/3640

DOI : 10.4000/assr.3640

ISSN : 1777-5825

Éditeur

Éditions de l'EHESS

Édition imprimée

Date de publication : 1 mai 2006

Pagination : 147-299

ISBN : 2-7132-2092-0

ISSN : 0335-5985

\section{Référence électronique}

Christian Décobert, "Gilles Veinstein, dir., Syncrétismes et hérésies dans l'Orient seldjoukide et ottoman (xive-xviiie siècles) », Archives de sciences sociales des religions [En ligne], 134 | avril - juin 2006, document 134-87, mis en ligne le 12 septembre 2006, consulté le 21 septembre 2020. URL : http:// journals.openedition.org/assr/3640 ; DOI : https://doi.org/10.4000/assr.3640

Ce document a été généré automatiquement le 21 septembre 2020

(C) Archives de sciences sociales des religions 


\title{
Gilles Veinstein, dir., Syncrétismes et hérésies dans l'Orient seldjoukide et ottoman (xive-xviiie siècles)
}

\author{
Louvain, Peeters, coll. « Turcica » (XIV), 2005, 428 p.
}

\section{Christian Décobert}

1 Il y a une valeur heuristique à parler du syncrétisme religieux. Voilà un concept inopérant pour cerner ce qui relève de l'appartenance du sujet croyant dans un groupement à caractère religieux, mais tout à fait démonstratif de la manière dont cette question a pu et est encore parfois abordée.

2 Il est né, comme opérateur savant, chez les spécialistes d'histoire religieuse, en particulier ceux qui traitent de l'Antiquité tardive (Michel Tardieu, «Les facettes du syncrétisme : méthodologie de la recherche et histoire des concepts ») pour décrire, notamment, les cultes orientaux à Rome, les dites religions à mystères, les phénomènes d'hénothéisme, c'est-à-dire les mouvements de transfert de culte en situation d'hétérogénéité culturelle, les phénomènes d'amalgame des dieux, de dénominations nouvelles. L'un des phénomènes considérés comme exemplaires du syncrétisme religieux étant celui des cultes isiaques, dont l'extraordinaire expansion était expliquée par le caractère composite et donc adapté à toutes les cultures concernées, puisque chacune pouvait, en quelque sorte, y reconnaître quelque chose d'elle-même ( $c f$. Françoise Dunand et Pierre Lévêque éds., Le syncrétisme dans les religions de l'Antiquité. Colloque de Besançon, 22-23 octobre 1973, EPRO 46, Leyde, 1975). Historiquement donc, le syncrétisme désignait, de façon privilégiée, les faits de circulation des dieux et d'inflation cultuelle qui précédèrent l'émergence et le triomphe du christianisme. Et il était instrumentalisé pour imposer l'idée que le christianisme précipitait l'abandon des syncrétismes, que l'appartenance chrétienne était une aversion de ces cultes composites et bigarrés, que le christianisme se bâtissait sur le principe de conversion et non d'adhésion, laquelle suppose une possible multiplicité d'adhésions (cf. Arthur Darby Nock, Conversion. The Old and the New in Religion from Alexander the Great to Augustine of Hippo, réimp. Baltimore-Londres, The Johns Hopkins University Press, 
1998 ; cf. Christian Décobert, «La conversion comme aversion », ASSR 104, 1998, p. 33-60). Au total, le concept de syncrétisme servait à décrire à la fois une préhistoire (la préhistoire chrétienne) et un modus operandi - la contamination qui emporte les systèmes religieux antiques, impose une multiplicité d'appartenances et préfigure sa propre fin dans le christianisme.

Mais le concept est flou, il s'étend aisément en performance, il perd rapidement en compétence - qui trop embrasse mal étreint. Dès ses premiers usages, il a été considéré comme inopérant, parce que décrivant tout et rien de spécifique : soit que toute préhistoire religieuse était syncrétisme (Joachim Wach, cité par M. Tardieu) ; soit qu'il était propre à toute expérience religieuse (G. van der Leeuw, cité par M. Tardieu) soit, plus largement, qu'il constituait le fait critique par essence, au cœur de tout questionnement de l'unité de l'être (Hans Jonas, cité par M. Tardieu). Pourtant, le concept de syncrétisme a été un succès renouvelé, grâce à l'anthropologie. Pour décrire, désormais, ce qui contrevient à la pureté que l'on veut décrire ou prescrire (André Mary, Le bricolage africain des héros chrétiens, Paris, Le Cerf, 2000). Pour signifier ce que l'on enregistre comme fait de contamination.

4 Parmi les «expériences» de syncrétismes en islam, le présent ouvrage traite prioritairement de ce qui fait syncrétisme entre l'islam et le chamanisme. À propos des Bektachis, notamment. Le réseau confrérique des Bektachis (né au XIII siècle, qui se développa considérablement dès le $\mathrm{XVI}^{\mathrm{e}}$ siècle et qui fut de grande puissance politique, puisque répandu chez les janissaires, et soutenu par le centre ottoman) était sunnite officiellement, mais attaché à la famille 'alide ('Alī, surtout) et donc soupçonné de contamination crypto-shī'ite. Mais il est également soupçonné de contamination par le chamanisme, dans la performance de certains rites mystiques (semah).

5 Or c'est bien dans ce dernier soupçon que se révèle, en quelque sorte, la « situation » du syncrétisme, qui articule la question de la contamination à la question du substrat symbolique ou religieux: les populations considérées, islamisées, pratiquaient le chamanisme avant l'islam, ou rencontraient le chamanisme dans une région où il était comme endémique. L'islam s'est installé en Asie centrale, une région où dominait la pratique chamanique. Mais l'on ne peut guère définir le chamanisme comme une religion, ainsi que les travaux de Roberte Hamayon l'ont clairement montré (Roberte Hamayon, La chasse à l'âme. Esquisse d'une théorie du chamanisme sibérien, Nanterre, Société d'ethnologie, 1990). Les chamanismes, au pluriel, sont un ensemble de pratiques de contact direct avec le suprasensible, caractérisées par une certaine gestuelle et par une pure oralité ; pratiques à vocation thérapeutique, les chamanismes n'en développent pas moins une véritable pensée symbolique.

6 Ce qui, en islam, apparaît comme contaminé par le chamanisme est la pratique mystique, le système des techniques de caractère "orgiastique ", pour user d'une qualifiant wébérien. C'est pourquoi l'on parle volontiers de «soufisme chamanisé » ou de "chamanisme soufisé" ( $c f$. Patrick Garrone, "Aperçu du chamanisme islamisé d'Asie centrale post-soviétique ", in Denise Aigle, Bénédicte Brac de la Perrière et JeanPierre Chaumeil éds., La politique des esprits, Nanterre, Société d'ethnologie, 2000 ; Thierry Zarcone, "Interpénétration du soufisme et du chamanisme dans l'aire turque. "Chamanisme soufisé" et "soufisme chamanisé" ", idem). Un excellent exemple est celui du semah : un terme soufi (samá') désignerait, en fait, une pratique de oyun, qui est une danse d'origine chamanique (Françoise Arnaud-Demir, « Le syncrétisme alévi-bektachi dans les chants accompagnant la danse rituelle semah »). Un processus de discrédit du 
oyun s'est produit, mais concomitant à une intégration de son contenu dans le bektachisme, ce qui a provoqué un recouvrement de sens par la terminologie soufie, d'où l'imposition du terme semah. Mais l'on peut comprendre ce qui se passe comme un processus formel d'hybridation, jouant sur deux niveaux de sens à la fois : le mi riāj comme voyage vers Dieu mais aussi comme rencontre des esprits ; le culte de 'Alī quasi divinisé, mais aussi le couple 'Alī et Fātima et la non discrimination sexuelle; le culte des saints 'alides mais aussi l'invocation d'intercession animale (la grue, notamment), etc. Il n'y a pas, à proprement parler, de réinterprétation de la dite tradition chamanique dans un système de sens islamique, il y a plutôt une combinatoire de sens et de symboliques restés distincts: immédiateté aux esprits - présence du couple homme-femme comme gage de fertilité - intercession animale; Dieu - présence de 'Alī comme gage de chaîne de salut - intercession des saints. L'on peut ainsi décrire ce qui se passe comme une superposition terme à terme de chacun des éléments posés en paradigme l'un de l'autre et comme une syntagmatique préservée par la mise en relation des éléments dans chacun des systèmes.

7 Certes, l'on évoquera, de façon extensive, le syncrétisme, mais ce terme n'étant que bien peu descriptif, il conviendra peut-être de parler d'hybridation et d'hybridité (cf. André Mary): ni fusion de sens, ni soumission systémique de l'une des pratiques (chamanique, islamique) à l'autre, mais combinatoire. Les contributions de Jean During ("Notes sur l'angélologie Ahl-e haqq»), d'Irène Mélikoff ("Le gnosticisme chez les Bektachis/Alévis et les interférences avec d'autres mouvements gnostiques»), ou de Yuri Stoyanov ( On Some Parallels between Anatolian and Balkan Heterodox Islamic and Christian Traditions and the Problem of their Coexistence and Interaction in the Ottoman Period »), permettent effectivement une telle lecture.

8 Mais l'essentiel est certainement ailleurs. André Mary distingue, fondamentalement, ce qui relève du syncrétisme comme processus et ce qui en relève comme politique, que cette politique soit " pro- » ou " anti-syncrétisme ». Or la question de la frontière entre islam et chamanisme ou entre islam et christianisme se rapporte au syncrétisme comme politique et non comme processus - lequel, encore une fois, est d'une banale universalité. Par exemple, quand on évoque les accusations de syncrétisme faites au bektachisme, de quelles accusations s'agit-il ? Et par qui ont-elles été exprimées? En d'autres termes, existe-t-il d'anciennes accusations de syncrétisme? Si oui, de quoi le bektachisme était-il réellement accusé, de quoi les Ahl-e haqq étaient-ils réellement soupçonnés ? D'hétérodoxie. Mais alors, de quoi parle-t-on réellement ? Le syncrétisme n'est certes pas une catégorie doctrinale. L'hétérodoxie (Yuri Stoyanov, op. cit.), le gnosticisme (Irène Mélikoff, op. cit.), le dualisme (Toufic Fahd, «Les sectes dualistes en terre d'Islam »), angélologie (Jean During, op. cit.) sont des notions savantes empruntées à une histoire générale des religions, à connotation chrétienne ou para-chrétienne (Jean Kellens, «Interprétations du dualisme mazdéen » qui décrit bien la trajectoire savante du terme " dualisme »), largement étrangères au cadre islamique de pensée. En revanche, ce qui touche aux questions de connaissance (connaissance de ce qui fait la vraie croyance), d'intermédiation (entre l'homme croyant et Dieu), d'unicité (de Dieu) relève bel et bien de la posture doctrinale en islam.

9 S'il est un anti-syncrétisme virulent, s'il est une hérésiographie, c'est sur l'irréfragable question de l'unicité divine, de la croyance en un Dieu unique et créateur de ce qui est. Les doctrines dualistes, les mouvements cabalistes furent répertoriés, dénoncés, si possible éradiqués. Le dualisme pensait un principe divin lié au Mal, comme un 
principe divin lié au Bien ; même édulcoré, dans la doctrine d'un principe mauvais qui aurait été vaincu, il niait l'absolu tawhịd. Le cabalisme, connu comme accordant un sens secret à ce qui est écrit (texte sacré, lettres) - ce qui n'est que l'une de ses manifestations -, pensait Dieu comme se développant, se disséminant infiniment, se manifestant par des émanations successives et donc engendrant toute chose de sa substance; il niait l'absolu distance de Dieu à l'objet de sa création. Il y a donc une hérésiographie, une littérature de la réfutation, une affaire de savants, qui ne doit certes pas tromper sur la réalité de tels mouvements hérésiarques en islam. Une telle littérature (Fahd) élaborait de véritables listes de mouvements religieux et de mouvements sectaires, elle balayait très large et traitait autant du passé que du présent, du manichéisme ancien que de tel microgroupe installé sensément dans telle région à telle époque. Mais l'on peut parfois douter de l'existence effective, comme groupes identifiés, organisés et durables, des mouvements sectaires qui sont mentionnés. L'objet d'une telle littérature est plus de couvrir le champ des possibles, quitte à forcer les identifications et à mettre une appellation sous une idée (une affirmation irrecevable en matière de dogme de l'unicité), qu'à consigner l'existant.

Au total, force est de constater que les frontières religieuses, quant à l'islam, ne sont pas les mêmes si l'on s'attache à la dogmatique ou si l'on s'attache à la pratique rituelle. La première ne souffrait aucune déviance; ou plutôt, elle se construisait en identifiant des déviances par rapport auxquelles le croyant avait à se situer. Pour paraphraser Howard Becker, disons qu'en matière de dogme, les hommes d'autorité créaient l'hérésie en instituant des normes dont la transgression constituait l'hérésie ( $c f$. Howard S. Becker, Outsiders. Études de sociologie de la déviance, Paris, Métailié, 1985). En l'occurrence, c'est la dogmatique de l'unicité divine qui produisait la vision du champ religieux comme clos. Rappelons-nous que l'islam s'était édifié, face essentiellement au christianisme, comme la religion de l'absolu de l'unicité divine. Que tout ce qui engageait aux violences contre les symboles chrétiens (la croix) se rapportait à cette affirmation quasi obsessive, que le premier monument islamique, le Dôme du Rocher de Jérusalem, revendiquait avec force - par ses inscriptions para-coraniques (cf. Oleg Grabar, «The Umayyad Dome of the Rock in Jerusalem », Ars Orientalis 3, 1959, p. 33-62) - la prééminence d'un abrahamisme nouveau qui, face aux autres monothéismes, affirmait le Dieu un et universel. Une religion construit notamment sa dogmatique en fonction de la manière dont, dans son âge premier, elle était apparue.

Quant à la pratique rituelle, en milieu soufi particulièrement, la posture partagée était tout autre. Rapidement dit, le soufisme confrérique a diffusé en islam une représentation de l'identité religieuse comme intime. Dans ce cadre, la pratique rituelle, qui en soi était intime (puisqu'elle engageait à une expérience de lien fusionnel de l'être croyant avec Dieu), pouvait tout à fait bien s'accommoder d'une hybridation, sans que la sincérité du croyant intime fût en quelque manière suspectée. Il convient, en effet, de ne pas confondre la motivation de l'acteur croyant et le contenu composite des gestes qu'il accomplit. L'humble bektachi qui pratiquait le semah - sachant qu'il s'agissait d'une technique extatique, en usant dans le but de se rapprocher de son Dieu et, ce faisant, invoquait les êtres qui Lui étaient proches ('Alī, notamment) - était bel et bien, pour lui-même et pour les autres, un musulman, vivant dans un cadre musulman. Que cette procédure fût également propitiatoire ou prophylactique ne contrevenait pas à la motivation de l'acte, au contraire, elle lui donnait force et légitimité. Les « éléments 
chamaniques » du semah produisaient un surplus de sens plutôt qu'un contresens ou une équivocité de sens.

Il y a, en conséquence, à prendre garde de ne pas combiner, même de façon contingente, les faits d'hybridation ou de syncrétisme et les faits de dissidence ou d'hérésie. Ces derniers sont les effets d'une politique de fermeture dogmatique. Les hybridations, comme nous l'avons vu pour le semah, jouent sur un tout autre registre : elles produisent une double syntagmatique de sens, de même qu'elles provoquent une ouverture de paradigme, puisqu'elles posent chacun des éléments face à un autre qui lui est équivalent et qui le recouvre. La question que formule explicitement le fait hérétique est celle de l'appartenance religieuse - l'hérésie n'existe que pour désigner les «non appartenants », elle fabrique symétriquement de l'appartenance et de la non appartenance. C'est précisément une question qui échappe à la pratique hybride, laquelle, dans un cadre religieux donné mais non discuté, fabrique ou renouvelle du sens.

13 En somme, si l'on veut comprendre comment s'exemplifiait l'idée d'appartenance, il suffit de se plonger dans la littérature hérésiographique, dont l'objet était de répertorier les déviances en matière d'unicité de Dieu, de connaissance de Dieu ou du message de Dieu, d'intermédiation entre l'homme et Dieu. À cet égard, l'œuvre de 'Abd al-Raḥmān al-Bistāmī est particulièrement éloquente (Denis Gril, «Ésotérisme contre hérésie : 'Abd al-Raḥmān al-Bistāmī, un représentant de la science des Lettres à Bursa dans la première moitié $\mathrm{du} \mathrm{Xv}^{\mathrm{e}}$ siècle $\left.»\right)$. Al-Bistāmī (1380-1454), qui vécut à Bursa (Anatolie) près de la cour ottomane, était un lettré et un mystique, appartenant à l'école d'Ibn 'Arabī, mais surtout connu pour son savoir ésotérique et sa science des Lettres. Dans son traité Al-Fawā'ih al-miskiyya fi l-fawātih al-makkiyya, il s'attacha notamment à circonscrire, parmi les voies de la connaissance, celles qui relevaient de l'orthodoxie et celles qui méritaient le discrédit de l'hérésie ou de l'imposture. Décrivant sa propre pratique de mystique et de savant des Lettres, Al-Bistāmī se faisait également hérésiographe.

Pour Al-Bistāmī, il y a bien une bonne et une mauvaise science des lettres. La bonne est celle qui affirme que les Lettres, puisqu'elles ont une valeur et qu'elles sont en relation avec les éléments naturels et les astres, sont une réalité intermédiaire dans l'au-delà et l'ici-bas. Par elles, la rencontre est possible avec le niveau supérieur de la réalité, celui des principes. Il est ainsi possible, par la science des Lettres et par leur mise en jeu (le carré magique, notamment) de faire agir ces principes supérieurs sur la réalité d'icibas, la réalité mondaine. La science des Lettres est à la fois une pratique de connaissant et une pratique propitiatoire ou prophylactique. Elle participe de l'héritage d'Ibn 'Arabī, qui est d'intégrer la connaissance dans la procédure d'intermédiation vers le monde des principes et de Dieu. La fausse science des Lettres mène à une fausse connaissance de Dieu : c'est celle qui engage les connaissants dans l'affirmation que des hommes, dont eux-mêmes, puissent être des manifestations divines et donc, à ce titre, agir sur les êtres et les choses. Et Al-Bistāmī de déclarer hérétiques les mouvements shīites, aussi bien que ceux qui se disent au-dessus des lois communes (les ibāhiyya, antinomistes), que nombre de réseaux confrériques et que les adeptes de la science «traditionnelle» des Lettres, les hurufiyya. C'est-à-dire les pensées de type émanationniste, qui suppose la dissémination divine de Dieu jusqu'à l'homme.

Encore une fois, la dichotomie s'établit clairement, entre le principe orthodoxe de l'intermédiation et la dérive hétérodoxe de l'émanation. Il s'agit là d'un construit 
doctrinal qui établit bel et bien une frontière d'appartenance. Il y a bien là un dedans et un dehors de l'islam, il y a bien un « entre nous » et un « autre que nous». 